\title{
COMPUTATIONAL COMPLEXITY OF SOME MAXIMUM AVERAGE WEIGHT PROBLEMS WITH PRECEDENCE CONSTRAINTS
}

\author{
ULRICH FAIGLE and WALTER KERN
}

\author{
University of Twente, Enschede, The Netherlands \\ (Received June 1991; revision received January 1993; accepted February 1993)
}

\begin{abstract}
Maximum average weight ideal problems in ordered sets arise from modeling variants of the investment problem and, in particular, learning problems in the context of concepts with tree-structured attributes in artificial intelligence. Similarly, trying to construct tests with high reliability leads to a nontrivial maximum average weight ideal problem. This paper investigates the computational complexity and shows that the general problem is NP-complete. Important special cases (e.g., finding rooted subtrees of maximal average weight), however, can be handled with efficient algorithms.
\end{abstract}

A ssume that $P$ represents a set of possible investments that are (partially) ordered by certain precedence constraints $i<j$ in the sense that investment $j$ may only be affected if investment $i$ is carried out as well. Let us, furthermore, assume that with each investment $i \in P$ there is associated its expected gain $r(i)$ and its cost $s(i)$. Then the investment problem with maximum yield asks to determine a feasible set $I \subseteq P$ of investments which maximizes the quotient $r(I) / s(I)$, i.e., the total expected gain relative to the total cost.

We will show in Section 2 that the investment problem outlined above may be solved in polynomial time. As pointed out by Haussler (1988), a variant of the investment problem also comes up in the context of "learning concepts with treestructured attributes" in artificial intelligence. In the latter case, our ordered set $\boldsymbol{P}$ is given by a rooted tree with the understanding that $i<j$ holds if $i$ lies on the (unique) path from $j$ to the root. Section 3 presents an algorithm for the maximum yield problem on rooted trees that is strongly polynomial in the size of the tree.

If one's budget for making investments is bounded by some limit $B \leqslant s(P)$, then one might be interested in maximizing the difference $r(I)-s(I)$, where $I$ runs over all feasible subsets $l$ of $P$ satisfying $s(I) \leqslant B$. This problem, however, includes the knapsack problem and thus is NP-complete (see Garey and Johnson 1979), which gives further evidence to the fact that solving maximum yield problems is easier for the rich than the poor. (Actually, the situation is not quite so bad: Johnson and Niemi (1983) derive pseudopolynomial algorithms that are particularly efficient for small values of $B$ ).

To formalize our computational model, we consider an arbitrary finite set $P$, partially ordered by precedence constraints. An ideal of $P$ is a feasible subset $I \subseteq P$ in the sense that $x \in I$ and $y<x$ in $P$ always implies $y \in I$. Relative to the given nonnegative functions $r: P \rightarrow \mathbb{Q}_{+}$and $s: P \rightarrow \mathbb{Q}_{+}$, the maximum average weight ideal problem is to maximize the quotient $r(I) / s(I)$, where $I$ runs over all ideals of $P$. Note that the choice $s \equiv 1$ yields the average weight in the usual sense: $\max r(I) /|I|$. By convention, we set $r(I) / s(I)=1$ if $r(I)=s(I)=0$, and $r(I) / s(I)=\infty$ if $r(I)>s(I)=0$. (Hence, in particular, $r(\phi) / s(\phi)=1)$.

An interesting extension of our model allows for the additional constraint of fixing the size of the feasible subsets of $P$ to $k$, say. We then obtain the cardinality restricted maximum average weight ideal problem, that is, to maximize the quotient $r(I) / s(I)$, where the maximum is taken over all feasible subsets $I$ of cardinality $k$. Let us illustrate how cardinality restricted problems naturally occur, for example, in the theory of optimal test construction (see Lord and Novik 1968 for details).

Assume that we are given a finite number of test items $i$ and, for each $i$, parameters $\sigma_{i}, \rho_{i} \geqslant 0$. We are to choose $N$ items so that the reliability of the resulting test is as large as possible. This amounts to solving the optimization problem:

Subject classifications: Analysis of algorithms: computational complexity. Networks/graphs: networks arising from precedence constraints. Program-
ming: integer programming and dynamic programming. Area of review: OPTIMIZATION. 
$\max \frac{\left(\sum \sigma_{\imath} \rho_{l} x_{\imath}\right)^{2}}{\sum \sigma_{\imath}^{2} x_{l}}$

such that $\sum x_{i}=N$, where $x_{i} \in\{0,1\}$.

In spite of the quadratic term in the objective function, we can model the problem as a cardinality restricted maximum average weight ideal problem on some ordered set $P$. For $P$, we consider all 1-element subsets $\{i\}$ and all 2-element subsets $\{i, j\}$ of test items, ordered by set-theoretic containment. Then we adjoin a new element 0 which is defined to be smaller than all the subsets above. We now choose $r, s: P \rightarrow$ Q as:

$$
\begin{aligned}
& \left\{\begin{array}{l}
r(0)=-M \cdot\left(\begin{array}{l}
N \\
2
\end{array}\right) \\
r\{i\}=\sigma_{\imath}^{2} \rho_{\imath}^{2} \\
r\{i, j\}=2 \sigma_{\imath} \rho_{\imath} \sigma_{\jmath} \rho_{\jmath}+M
\end{array}\right. \\
& \begin{cases}s(0)=0 \\
s\{i\}=\sigma_{\imath}^{2} \\
s\{i, j\}=0 .\end{cases}
\end{aligned}
$$

Let $k=1+N+\left(\begin{array}{c}N \\ 2\end{array}\right)$ and let $I$ be an ideal in $P$ of cardinality $|I|=k$. Observe that $I$ includes at most $\left(\begin{array}{c}N \\ 2\end{array}\right)$ 2-element subsets $\{i, j\}$ of items (otherwise we would have $|I|>k$ ). Hence, if $M>0$ is large enough, we conclude that $r(I) \geqslant 0$ if and only if $I$ contains exactly $N$ singletons. Consequently, $r(I) \geqslant 0$ implies that $\{i, j\} \in I$ must hold whenever both $\{i\},\{j\} \in I$. In other words, $I$ yields a feasible solution for the test construction problem. Moreover, $I$ is of maximum average weight if and only if $I$ maximizes the objective function in $(*)$.

\section{AN NP-COMPLETENESS RESULT}

In this section, we show that the decision problem associated with the maximum weight problem with cardinality constraints (cf. problem 3 in the Introduction) is NP-complete for orders of height 1 (i.e., for orders whose Hasse diagram is a bipartite graph). The same construction also reveals the problem of minimizing a submodular set function over all subsets of given cardinality $k$ to be NP-hard.

Theorem 1. The following problem is NP-complete:

Instance: An ordered set $P$, a weighting $r: P \rightarrow \mathbb{Z}+$ and integers $k, R \in \mathbb{Z}_{+} \backslash\{0\}$.

Question: Does there exist an ideal $I$ in $P$ with $|I|=$ $k$ and $r(I) \geqslant R$ ?

Proof. We use reduction from MAXIMUM CLIQUE. Let $G=(V, E)$ be a graph with sets $V$ of vertices and $E$ of edges. We define the order $P$ on $V \cup E$ via $v<e$ if $v \in V$ is an endpoint of $e \in E$. The ideals of $P$ are thus of the form $E^{\prime} \cup V^{\prime}$, where $E^{\prime} \subseteq E$ and $V^{\prime} \subseteq V$ contains all the endpoints of the edges in $E^{\prime}$. Consider $r: P \rightarrow \mathbb{Z}_{+}$given by

$r(x)=\left\{\begin{array}{lll}0 & \text { if } & x \in V \\ 1 & \text { if } \quad x \in E .\end{array}\right.$

Claim. $G$ contains a clique of size $s$ if and only if $P$ contains an ideal $I$ with $|I|=\left(\begin{array}{l}s \\ 2\end{array}\right)+s$ and $r(I) \geqslant\left(\begin{array}{l}s \\ 2\end{array}\right)$.

To see that the claim holds, note first that any clique $C$ of size $s$ in $G$ gives rise to an ideal $I$ with the desired properties by letting $I$ consist of all edges and vertices of $C$. Conversely, let $I=E^{\prime} \cup V^{\prime}$ be an ideal in $P$ with $|I|=\left(\begin{array}{l}s \\ 2\end{array}\right)+s$ and $r(I)=\left|E^{\prime}\right| \geqslant\left(\begin{array}{l}s \\ 2\end{array}\right)$. Now the definition of $P$ implies for $i=\left|V^{\prime}\right|$ that $\left|E^{\prime}\right| \leqslant\left(\begin{array}{l}x \\ 2\end{array}\right)$. Hence $|I|=\left|E^{\prime}\right|+\left|V^{\prime}\right|$ allows us to conclude that $i \geqslant s$. If $i>s$, then $|I|=\left(\begin{array}{l}s \\ 2\end{array}\right)+s$ yields $\left|E^{\prime}\right|<\left(\begin{array}{l}s \\ 2\end{array}\right)$. So we must have that $\left|V^{\prime}\right|=s$ and $\left|E^{\prime}\right|=\left(\begin{array}{l}s \\ 2\end{array}\right)$, i.e., $E^{\prime}$ and $V^{\prime}$ correspond to a clique in $G$ of size $s$.

Similarly, we observe that the decision problem associated with the corresponding minimum weight problem is NP-complete:

Instance: An ordered set $P$, a weighting $r: P \rightarrow \mathbb{Z}_{+}$ and integers $k, R \in \mathbb{Z}_{+} \backslash\{0\}$.

Question: Does there exist an ideal $I$ in $P$ with $|I|=$ $k$ and $r(I) \leqslant R$ ?

For the reduction from MAXIMUM CLIQUE, we consider the same ordered set $P$ as in the proof of Theorem 1 endowed with the weighting $\bar{r}: P \rightarrow \mathbb{Z}_{+}$:

$\bar{r}(x)= \begin{cases}1 & \text { if } \quad x \in V \\ 0 & \text { if } \quad x \in E .\end{cases}$

It is then straightforward to verify that $G$ contains a clique of size $s$ if and only if $P$ contains some ideal $I$ with $|I|=\left(\begin{array}{l}s \\ 2\end{array}\right)+s$ and $\bar{r}(I) \leqslant s$.

The weight function $r$ (and $\bar{r}$ ) considered above is modular in the sense that $r$ satisfies

$r(A \cup B)+r(A \cap B)=r(A)+r(B)$

for all ideals $A, B \subseteq P$. In the case where $P$ is trivially ordered (and hence every subset is an ideal of $P$ ), a subset of given cardinality and maximal or minimal $r$-weight can easily be found by the greedy algorithm in polynomial time because modular functions are additive. This is no longer true for submodular set functions.

Recall that $f: 2^{s} \rightarrow \mathbb{Z}$, where $S$ is an (unordered) set, is submodular if for all $A, B \subseteq S$,

$f(A \cup B)+f(A \cap B) \leqslant f(A)+f(B)$. 
It is known that a subset minimizing the submodular set function $f$ can be found in polynomial time via the ellipsoid algorithm (cf. Götschel, Lovász and Schrijver 1981). For the cardinality-restricted minimization problem, however, we obtain an NP-hardness result.

Proposition 1. The following problem is NP-hard:

Instance: $A$ set $S$, a submodular function $f: 2^{s} \rightarrow \mathbb{Z}_{+}$ and integers $k, R \in \mathbb{Z}_{+}$.

Question: Does there exist a subset $A$ of $S$ with $|A|=$ $k$ and $f(A) \leqslant R$ ?

Proof. To see that our problem is at least as hard as MAXIMUM CLIQUE, we consider a graph $G=$ $(V, E)$ and define for each $E^{\prime} \subseteq E: f\left(E^{\prime}\right)=\left|V\left(E^{\prime}\right)\right|$, where $V\left(E^{\prime}\right)$ is the set of endpoints of edges in $E^{\prime}$. It is easy to verify that $f$ is submodular. Moreover, $G$ contains a clique of size $c$ if and only if $E$ has a subset $A \subseteq E$ with $|A|=\left(\begin{array}{l}c \\ 2\end{array}\right)$ and $f(A) \leqslant c$.

\section{DROPPING THE CARDINALITY CONSTRAINT}

We now show that the maximum weight ideal problem becomes polynomial when the cardinality constraint is dropped. As a consequence, the maximum average weight problem can be seen to be polynomial.

Lemma 1. Let $P$ be an ordered set and $w: P \rightarrow \mathbb{Q} a$ (not necessarily nonnegative) weight function. Then the problem max $w(I)$ such that I ideal of $P$ can be solved in polynomial time.

Proof. We write the problem as an integer linear program:

$\max \sum_{i \in P} w(i) x(i)$

such that

$$
\begin{array}{rlrl}
x(i)-x(j) & \geqslant 0 \quad \text { for all } i<j \text { in } P \\
0 & \leqslant x \leqslant 1 & \\
x(i) & \in\{0,1\} \quad \text { for all } i \in P .
\end{array}
$$

The constraint matrix of this problem essentially is the (transpose of) the node-arc incidence matrix of the directed comparability graph of $P$. Hence, it is clear that we have a totally unimodular constraint matrix. It follows that we may drop the integrality constraints and obtain an LP-relaxation with integral optimal solutions, which solves the original problem.

Note that Lemma 1 may be sharpened to include the maximization problem over all nonempty ideals of
$P$. Indeed, for any given $i_{0} \in P$, we may add the constraint $x\left(i_{0}\right)=1$ to the linear program and still retain total unimodularity. Hence, we have to solve at most $|\boldsymbol{P}|$ LPs to obtain a solution for the modified maximization problem.

We remark that the optimization problem in Lemma 1 can be reduced to a network flow problem via a construction due to Picard (1976) that essentially consists of considering the associated dual linear program. We define a network $G$ whose nodes are the elements of $P$ together with a source $u$ and a sink $v$. The edges of $G$ are of the form $(i, j)$ for $i>j$ in $P$, $(u, i)$ and $(i, v)$ for $i \in P$. The nonzero capacities are

$$
\begin{array}{ll}
c(i, j)=\infty & \text { if } i>j \text { in } P \\
c(u, i)=w(i) & \text { if } w(i)>0 \\
c(i, v)=-w(i) & \text { if } w(i)<0 .
\end{array}
$$

Let $(U, V)$ be a cut in $G$ with $u \in U$ and $v \in V$. Then the capacity $c(U, V)$ is finite if and only if $U \backslash\{u\}$ is an ideal in $P$. For such a cut $(U, V)$, we have

$$
\begin{aligned}
c(U, V) & =\sum_{\substack{w(i)<0 \\
l \in U \backslash u\}}}-w(i)+\sum_{\substack{w(l)>0 \\
l \in V \backslash\{v\}}} w(i) \\
& =-w(U \backslash\{u\})+\sum_{\substack{w(l)>0 \\
i \in P}} w(i) .
\end{aligned}
$$

Hence, $c(U, V)$ is minimal if and only if $w(U \backslash\{u\})$ is maximal, i.e., the problem is reduced to a minimal cut computation in a capacitated network.

Theorem 2. Let $r, s: P \rightarrow \mathbb{Z}_{+}$be two nonnegative weight functions. Then the problem

$\max \frac{r(I)}{s(I)}$ such that I is a nonempty ideal of $P$

can be solved in polynomial time.

Proof. We first check whether the maximum is infinite or not. According to our conventions (see the Introduction), the maximum is infinite if and only if there is some ideal with $s(I)=0$ and $r(I)>0$. This is easy to decide by looking at the unique (!) maximal $I_{0}$, say, with $s$-weight equal to zero. If $r\left(I_{0}\right)>0$, the maximum is infinite; otherwise it is not.

Assume next that the maximum is finite:

$q^{*}:=\max \frac{r(I)}{s(I)}<\infty$.

Now obviously $q^{*} \leqslant r(P)$. Moreover, for any $q>0$, we can decide in polynomial time whether there exists a nonempty ideal $I$ satisfying $r(I) / s(I)>q$. This follows by applying Lemma 1 to the weight function $w(i)=r(i)-q s(i)$ for all $i \in P$, 
as $w(I)>0$ implies $r(I) / s(I)>q$.

The final observation is that any two nonempty ideals $I_{1}$ and $I_{2}$ of $P$ satisfy either

$\frac{r\left(I_{1}\right)}{s\left(I_{1}\right)}=\frac{r\left(I_{2}\right)}{s\left(I_{2}\right)}$ or $\left|\frac{r\left(I_{1}\right)}{s\left(I_{1}\right)}-\frac{r\left(I_{2}\right)}{s\left(I_{2}\right)}\right| \geqslant[s(P)]^{-2}$.

Hence, we may perform binary search over the interval $[0, r(P)]$ and find an optimal ideal after at most $\left\lceil\log _{2} r(P)\right\rceil+\left\lceil 2 \log _{2} s(P)\right\rceil$ probes.

We remark that the assumption of integral $r$ - and $s$-values is not essential for Theorem 2 . In the case of arbitrary rational numbers, one may obtain an equivalent integral problem by scaling all weights by a factor $\lambda$, where $\lambda$ is the product of all denominators occurring in the $r(i)$ 's and $s(i)$ 's.

We stress the fact, however, that the nonnegativity assumption-at least for the weight function $s$-is necessary. Indeed, if $r \equiv 1$ on the (unordered) set $P$, then the maximum is infinite if and only if there is a subset $I \subseteq P$ with $s(I)=0$ and $I \neq \phi$. Using reduction from SUBSET SUM, the latter is easily seen to be a generally NP-complete decision problem.

A generalization of Theorem 2 in the framework of submodular functions can be obtained as follows. Let $\mathscr{F}=\mathscr{F}(P)$ be the collection of all ideals of $P$ and consider two functions $f, g: \mathscr{F} \rightarrow \mathbb{Z}_{+}$such that $g$ and $(-f)$ are submodular with $f(\phi)=0$ and $g(I)=0$ exactly when $I=\phi$. Then the problem

$\max _{I \in \mathscr{F}} \frac{f(i)}{g(i)}$

may be solved as in the proof of Theorem 2, provided we know an upper bound for $f(I)$ and for $g(I)$. This is so because the function $q \cdot g(I)-f(I)$ is submodular on $\mathscr{F}$ when $q \geqslant 0$, and hence can be minimized via the ellipsoid algorithm (cf. Grötschel, Lovász and Schrijver).

\section{ROOTED TREES}

Let us turn our attention to a rooted tree $T$, the root of which we will denote by $v_{0}$. Our first result says that maximal weight subtrees rooted at $v_{0}$ can be found in polynomial time even when the cardinality is prescribed (Theorem 3 ). We need some notation.

We order $T$ via the relation $u \leqslant v$ if the node $u$ lies on the unique path from $v_{0}$ to $v$ in $T$. Thus, the ideals relative to this order are exactly the subtrees rooted at $v_{0}$. Let $v_{1}, v_{2}, \ldots, v_{t}$ be the sons of $v_{0}$ in $T$. Then for $i=0,1, \ldots, t, T_{t}=\left\{v \in T: v_{t} \leqslant v\right\}$ is a subtree of $T$ rooted at $v_{l}$. Let the symbol $T_{\imath}^{\prime}$ stand for a subtree of $T_{\imath}$ rooted at $v_{l}$.
Theorem 3. Let $T$ be a rooted tree and $w: T \rightarrow \mathbb{Q} a$ (not necessarily nonnegative) weight function. Then for each $k \in \mathbb{Z}_{+}, \max \left\{w\left(T_{0}^{\prime}\right):\left|T_{0}^{\prime}\right|=k\right\}$ can be found in $O\left(n^{4}\right)$ steps, where $n=|T|$.

Proof. For every $0 \leqslant \ell \leqslant\left|T_{\imath}\right|$, we define

$w\left(\ell, T_{\imath}\right):=\max \left\{w\left(T_{\imath}^{\prime}\right):\left|T_{\imath}^{\prime}\right|=\ell\right\}$.

Furthermore, for $1 \leqslant i \leqslant t$ and $0 \leqslant \ell \leqslant\left|T_{1}\right|+\left|T_{2}\right|$ $+\cdots+\left|T_{1}\right|$, we set

$w(\ell, i):=\max \left\{w\left(T_{1}^{\prime}\right)+\cdots+w\left(T_{l}^{\prime}\right): \sum_{j=1}^{l}\left|T_{\jmath}^{\prime}\right|=\ell\right\}$.

Note that the $w(\ell, i)$ 's are easy to compute from the $w\left(\ell^{\prime}, T_{\iota}\right)$ 's $\left(\ell^{\prime} \leqslant \ell\right)$ :

$$
\begin{gathered}
w(\ell, 1)=w\left(\ell, T_{1}\right) \\
w(\ell, i+1)=\max \left\{w\left(\ell^{\prime}, i\right)+w\left(\ell^{\prime \prime}, T_{\imath+1}\right)\right. \\
\left.: \ell=\ell^{\prime}+\ell^{\prime \prime}\right\} .
\end{gathered}
$$

The solution of our original problem is given by

$w\left(k, T_{0}\right)=w\left(v_{0}\right)+w(k-1, t)$.

This suggests the following recursive ("bottom up") algorithm for determining $w\left(k, T_{0}\right)$ : Starting with the leaves of $T$, we successively move up to the root $v_{0}$ by computing $w\left(\ell, T^{\prime}\right)$ for larger and larger rooted subtrees $T^{\prime}$ of $T$.

Since no more than $n$ steps are needed to compute $w(\ell, i+1)$ from the values obtained for previous subtrees, each node of $T$ can be dealt with in $O\left(n^{3}\right)$ operations. Hence, we obtain the overall bound $\mathrm{O}\left(n^{4}\right)$.

Corollary 1. Let $r, s: T \rightarrow \mathbb{Z}_{+}$be two nonnegative weight functions and $k \in \mathbb{Z}_{+}$. Then the problem

$\max \frac{r\left(T_{0}^{\prime}\right)}{s\left(T_{0}^{\prime}\right)}$ such that $\left|T_{0}^{\prime}\right|=k$

is polynomially solvable.

Proof. We proceed as in the proof of Theorem 2 and solve the problem

$\max w_{q}\left(T_{0}^{\prime}\right) \quad$ such that $\left|T_{0}^{\prime}\right|=k$,

where $w_{q}(v)=r(v)-q \cdot s(v)$ for each $v \in T$, for different values of $q$ while performing binary search.

Given the tree $T$ with root $v_{0}$, the unrestricted problem

$\max \frac{r\left(T_{0}^{\prime}\right)}{s\left(T_{0}^{\prime}\right)}$, 
which was considered in Haussler, can be solved either by solving the problem in Corollary 1 for $k=$ $1, \ldots, n$ or by applying the algorithm from Theorem 2. In either case, binary search makes the number of steps carried out dependent on the sizes of the $r$ - and $s$-weights. It may, therefore, be interesting to point out that the unrestricted average weight problem on trees is strongly polynomial in the sense that it can be solved by an algorithm whose number of operations is bounded by a polynomial in $n$ (assuming that arithmetic operations can be carried out in unit time).

Theorem 4. Let $T$ be a tree with root $v_{0}$ and $r, s: T \rightarrow$ $\mathbb{Q}_{+}$be weight functions. Then the problem

$\max \frac{r\left(T_{0}^{\prime}\right)}{s\left(T_{0}^{\prime}\right)}$

is strongly polynomial.

Proof. For each node $v \in T$, we choose an optimal solution $T^{*}(v)$ to the problem

$\max \frac{r\left(T^{\prime}(v)\right)}{s\left(T^{\prime}(v)\right)}$,

where $T^{\prime}(v)$ runs over all subtrees rooted at $v$ (including the empty tree). Furthermore, we set

$q^{*}(v):=\frac{r\left(T^{*}(v)\right)}{s\left(T^{*}(v)\right)}$

Suppose that we already know optimal solutions $T^{*}(v)$ for all $v \neq v_{0}$. Then algorithm $\mathbf{A}$ computes an optimal $T^{*}\left(v_{0}\right)$ as follows, where $N\left(T^{*}\right)$ denotes the set of nodes in $T \backslash T^{*}$ that are adjacent to some node in $T^{*}$.

\section{Algorithm A}

INIT: $T^{*}:=\left\{v_{0}\right\}$

LOOP: IF $N\left(T^{*}\right)=\phi$, THEN GOTO EMPTY

ELSE choose $v^{*} \in N\left(T^{*}\right)$ with $q^{*}\left(v^{*}\right)$ maximal

IF $q^{*}\left(v^{*}\right)<r\left(T^{*}\right) / s\left(T^{*}\right)$, THEN GOTO EMPTY

ELSE $T^{*}:=T^{*} \cup\left\{v^{*}\right\}$ GOTO LOOP

EMPTY: IF $r\left(T^{*}\right) / s\left(T^{*}\right)<1$, THEN $T^{*}:=\phi$ END.

Note that algorithm $\mathbf{A}$ includes the empty tree as a possible optimal solution. The algorithm is easy to adapt when one is to maximize over all nonempty $T_{0}^{\prime}$.

Let us give a brief sketch of the proof of correctness for algorithm $\mathbf{A}$. We proceed by induction on $n=|T|$. For $n=1$, there is nothing to show. The inductive step essentially reduces to the verification of the following claim:

If $v_{1}, \ldots, v_{t}$ with $q^{*}\left(v_{1}\right) \geqslant \cdots \geqslant q^{*}\left(v_{t}\right)$ are the sons of the root $v_{0}$ in $T$ and $q^{*}\left(v_{1}\right) \geqslant r\left(v_{0}\right) / s\left(v_{0}\right)$, then there is a tree $T_{0}^{*}$ rooted at $v_{0}$ such that $v_{1} \in T_{0}^{*}$ and $T_{0}^{*}$ is an optimal solution for

$\max _{T_{0}^{\prime} \neq \phi} \frac{r\left(T_{0}^{\prime}\right)}{s\left(T_{0}^{\prime}\right)}$

Moreover, if $q^{*}\left(v_{1}\right)<r\left(v_{0}\right) / s\left(v_{0}\right)$, then no $T_{0}^{\prime}$ containing $v_{1}$ is optimal.

The claim is a consequence of the implication

$\frac{a}{b} \leqslant \frac{c}{d} \Rightarrow \frac{a}{b} \leqslant \frac{a+c}{b+d} \leqslant \frac{c}{d}$

for all $a, b, c, d>0$. Indeed, if

$T_{0}^{\prime}=\left\{v_{0}\right\} \cup T_{2}^{\prime} \cup \cdots \cup T_{t}^{\prime}$

is an arbitrary rooted subtree not containing $v_{1}$, we conclude that

$q\left(T_{0}^{\prime}\right) \leqslant \max \left\{\frac{r\left(v_{0}\right)}{s\left(v_{0}\right)}, \frac{r\left(T_{2}^{\prime}\right)}{s\left(T_{2}^{\prime}\right)}, \ldots, \frac{r\left(T_{t}^{\prime}\right)}{s\left(T_{t}^{\prime}\right)}\right\} \leqslant q^{*}\left(v_{1}\right)$

and, therefore,

$q\left(T_{0}^{\prime} \cup T^{*}\left(v_{1}\right)\right)=\frac{r\left(T_{0}^{\prime}\right)+r\left(T^{*}\left(v_{1}\right)\right)}{s\left(T_{0}^{\prime}\right)+s\left(T^{*}\left(v_{1}\right)\right)} \geqslant q\left(T_{0}^{\prime}\right)$.

It is important to note that algorithm $\mathbf{A}$ adds only the node $v^{*}$ and not the tree $T^{*}\left(v^{*}\right)$ to the tree $T^{*}$ in each iteration of the loop. This is so because of the basic nontransitivity in $\mathbb{Q}$ :

$\frac{a}{b} \leqslant \frac{c}{d} \Rightarrow \frac{x+a}{y+b} \leqslant \frac{x+c}{y+d}$.

The number of steps in algorithm $\mathbf{A}$ is not more than $\mathrm{O}\left(n^{2}\right)$. Hence, the maximization problem

$\max \frac{r\left(T_{0}^{\prime}\right)}{s\left(T_{0}^{\prime}\right)}$

can be solved in $O\left(n^{3}\right)$ steps by applying algorithm $\mathbf{A}$ recursively ("bottom up") to every node of $T$.

We close this section with a remark on the LPrelaxation of the problem in Theorem 3 . That is, we consider the linear program

$\max \sum_{i \in T} w(i) x(i)$

such that $x(i)-x(j) \leqslant 0$ for all $i \leqslant j$ in $T$

$\sum_{i \in T} x(i)=k$

$0 \leqslant x \leqslant 1$.

In contrast to the LP-relaxation in the proof of Lemma 1 this linear program does not necessarily 


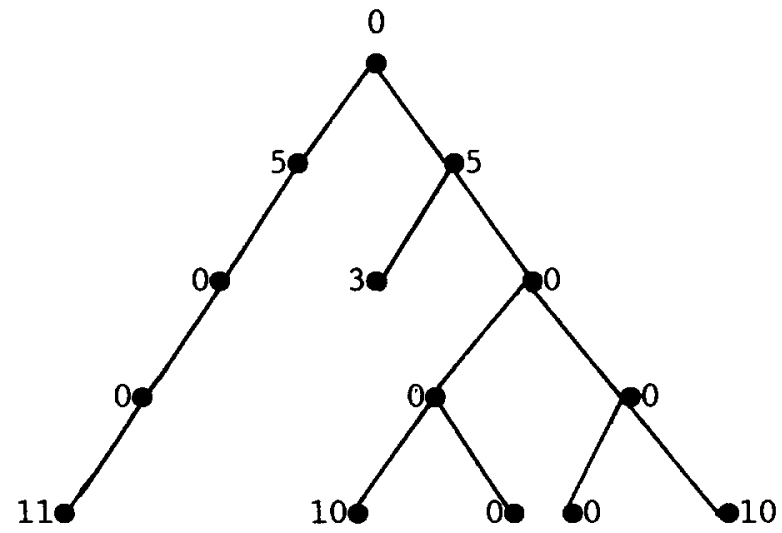

Figure 1. Weights $w(i)$.

have integral optimal solutions (cf. the example in the figures). Nevertheless, it is not hard to see that optimal solutions can be given in a particularly structured form: If $x^{*}$ is a basic optimal solution, then there exist subtrees $T^{\prime}$ and $T^{\prime \prime}$ of $T$ such that

$$
\begin{cases}x^{*} \equiv 1 & \text { on } T^{\prime} \\ x^{*} \equiv \text { const } & \text { on } T^{\prime \prime} \\ x^{*} \equiv 0 & \text { elsewhere }\end{cases}
$$

An example is given in Figures 1 and 2.

\section{REMARKS AND OPEN PROBLEMS}

The polynomiality results derived for rooted trees in Section 3 allow a straightforward extension to socalled series-parallel ordered sets. Are there more general classes of orders for which the cardinality restricted maximum weight ideal problem is polynomially solvable? Is the cardinality restricted maximum average weight problem (cf. Corollary 1) strongly polynomial on rooted trees?

Is it possible to characterize the solutions for the LP-relaxation of the cardinality restricted maximum weight problem (cf. the example in Section 3) under more general order constraints? Is the test construction problem (see the Introduction) polynomial? Is the general unrestricted maximum average weight ideal problem strongly polynomial?

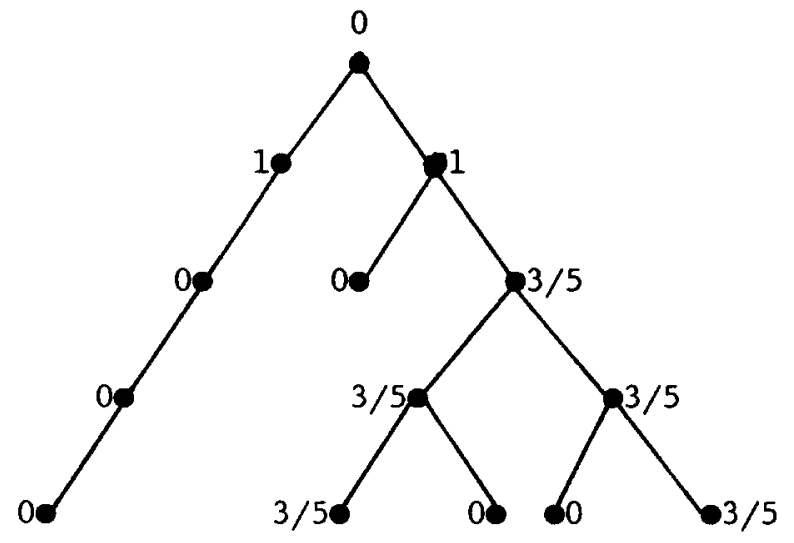

Figure 2. Optimal solution $x^{*}$ for $k=6$.

\section{NOTE ADDED IN PROOF}

A. Tamir pointed out that the restricted average weight problem on rooted trees and the general unrestricted average weight problem can be solved in strongly polynomial time by applying the scheme of Meggido (MOR 4 1979, 414-424) to the parametric optimization problem.

\section{REFERENCES}

GaReY, M. R., AND D. S. Johnson. 1979. Computers and Intractability. A Guide to the Theory of NP. Completeness. Freeman and Co., San Francisco.

GrotsChel, M., L. LoVÁsz AND A. SChriJVer. 1981. The Ellipsoid Method and its Consequences in Combinatorial Optimization. Combinatorica 1, 169-197.

Haussler, D. 1988. Quantifying Inductive Bias: AI Learning Algorithms and Valiant's Learning Framework. Artif. Intell. 36, 177-221.

Johnson, D. S., AND K. A. NiEMI. 1983. On Knapsacks, Partitions, and a New Dynamic Programming Technique for Trees. Math. OR 8, 1-14.

LORD, F. M., AND M. R. NoviK. 1968. Statistical Theories of Mental Test Scores. Addison-Wesley, Reading, Mass.

PICARD, J.-C. 1976. Maximal Closure of a Graph and Applications to Combinatorial Problems. Mgmt. Sci. 22, 1268-1272. 
Copyright 1994, by INFORMS, all rights reserved. Copyright of Operations Research is the property of INFORMS: Institute for Operations Research and its content may not be copied or emailed to multiple sites or posted to a listserv without the copyright holder's express written permission. However, users may print, download, or email articles for individual use. 